in vivo $36: 501-509$ (2022)

doi:10.21873/invivo.12731

\title{
Sternocleidomastoid Muscle Transfer for Treatment of Longstanding Facial Paralysis: Long-term Outcomes and Complications
}

\author{
WENJIN WANG $^{1 *}$, YIZUO CAI $^{1 *}$, CARLO M. ORANGES ${ }^{2}$, \\ DANIEL F. KALBERMATTEN ${ }^{2}$, DIRK J. SCHAEFER ${ }^{3}, \mathrm{CHUAN}$ YANG $^{1}$ and WEI LI ${ }^{1}$ \\ ${ }^{1}$ Plastic and Reconstructive Surgery Department, Shanghai Ninth People's Hospital, \\ Shanghai Jiao Tong University School of Medicine, Shanghai, P.R. China; \\ ${ }^{2}$ Department of Plastic, Reconstructive and Aesthetic Surgery, \\ Geneva University Hospitals, University of Geneva, Geneva, Switzerland; \\ ${ }^{3}$ Department of Plastic, Reconstructive, Aesthetic and Hand Surgery, \\ Basel University Hospital, University of Basel, Basel, Switzerland
}

\begin{abstract}
Background/Aim: The use of sternocleidomastoid muscle (SCM) flap for facial reanimation was established in the 1980s by the senior author of this paper. We aimed to analyze long-term outcome and complications of this procedure. Patients and Methods: We conducted a retrospective chart review of all patients undergoing SCM reanimation for longstanding facial palsy between January 2009 and December 2015. Patients with follow-up longer than 12 months (range=12-96) were included in the study. Facial muscle function was evaluated before and at each follow-up after the surgery with the House-Brackmann (HB) scale-facial nerve grading system and Facegram analysis. Donor site morbidity and overall complication rates were documented and analyzed. Results: Forty-two patients aged 18-66 years (mean age $=37$ ) with a mean duration of facial
\end{abstract}

This article is freely accessible online.

*These Authors contributed equally to this work and are listed as co-first authors.

Correspondence to: Wei Li, MD, Ph.D., Plastic and Reconstructive Surgery Department, Shanghai Ninth People's Hospital, Shanghai Jiao Tong University School of Medicine, 200011, Shanghai, P.R. China. Tel: +86 2123271699-5122, Fax: +86 2123271699, e-mail: liweiboshi@163.com; or Chuang Yang, MD, Ph.D., Plastic and Reconstructive Surgery Department, Shanghai Ninth People's Hospital, Shanghai Jiao Tong University School of Medicine, 200011, Shanghai, P.R. China. Tel: +86 2123271699-5122, Fax: +86 2123271699, e-mail: Patrick-wt@outlook.com

Key Words: Facial reanimation, sternocleidomastoid muscle, facial nerve, local flap, facial palsy. palsy of 5 years (range $=2-48$ ) met the inclusion criteria. The $H B$ score 2 years after surgery improved significantly $(p<0.05)$ in comparison to the pre-operative condition (3.6 vs. 4.7). Twelve months after surgery, oral commissure excursion improved by mean $8.95 \mathrm{~mm}$. No flap necrosis occurred, nor compromise of neck and shoulder function despite an obvious contour defect in the SCM donor site. None of the patients presented head posture or movement issues. Conclusion: The SCM flap transfer is a reliable and effective procedure to achieve moderate improvement of the oral commissure excursion using a local method with moderate donor site morbidity. It can be regarded as a valuable option for dynamic facial reanimation in case of longstanding facial palsy.

Facial paralysis can be caused by various insults to the facial nerve including trauma, acoustic neuroma, inflammation or virus infection (Ramsy Hunt syndrome) (1). The consequences, both psychologically and physically, are devastating for the patients as it results in progressive functional compromise of facial expression control and related serious social embarrassment (2-4).

Either direct anastomosis of the severed facial nerve or nerve transfer of branches of other cranial nerves (V, XI, or XII) can be applied to reactivate the paralyzed mimetic muscles when they are still viable (5-9). However, in case of longstanding facial palsy, in which the mimetic muscles have already gone through fibrosis and have therefore lost their ability to be reinnervated, local or free muscle flap transfer are needed to achieve a dynamic reconstruction (10-13). Free muscle flaps have been preferred by most surgeons as they are superior to local muscle flaps in terms of muscle force and, thus, improve oral commissure excursion in most cases (14). 
However, they require adequate donor vessels and nerves and their use is impossible if the recipient vessels are missing or unreliable, which is not uncommon in patients who had previously undergone extensive surgeries or radiation therapy in the recipient site. In this situation, pedicle muscle flap transfer may be used as an easy and reliable alternative.

Currently, the temporalis muscle is the most frequently used local muscle flap. Indeed, it provides good resting tone to the affected side of the oral commissure and enables the patients to generate a moderate but limited smile on clenching $(10,11,15)$. However, the proper functioning of this flap depends on a normal function of the trigeminal nerve. In particular, patients with a concomitant injury of the mandibular branch of the trigeminal nerve (V3) should not be treated with this procedure.

Our group previously described the use of the sternocleidomastoid muscle (SMC) transposition as alternative local muscle flap for facial reanimation (16). The SMC flap was first described by Jianu in 1882 (17). Overall, the flap presents the advantage of: i) rich vascularization, ii) possible use as combined flap, iii) possible shifting of the two heads of the muscle, and iv) providing favorable aesthetic results (18). Therefore, since then, the SCM flap has been widely used in head and neck reconstruction to cover the defects of the floor of the mouth, pharynx, and mandible as well as defects post-parotidectomy or due to Frey syndrome $(19,20)$. Despite its general popularity in head and neck reconstruction, there have been only few descriptions of the successful use of this flap for the reanimation of facial palsy so far (16). One of the authors of this paper (C.Y.) first developed the technique to reanimate facial palsy with this flap in our center and performed 321 cases since 1980s. Here, we share our experience with this procedure investigating effectiveness, long-term outcomes and possible complications.

\section{Patients and Methods}

Patients. We performed a retrospective review chart to include all patients with long-standing facial palsy or frozen faces treated in our center with SCM transfer for facial reanimation. All patients treated between 2009 and 2015 were screened, since after 2009 the procedure was standardized in 2009 and and remained unchanged thereafter. Only patients with follow-up longer than 12 months were included. Demographics of the patients, details of the anatomy, functional outcomes and complications were collected and analyzed. Details of the surgical technique, postoperative treatment and methods of evaluation and statistical analysis are reported below in separate paragraphs. All procedures performed in this study were in accordance with the ethical standards of the institutional and national research committee and with the 1964 Helsinki declaration and its later amendments or comparable ethical standards.

Surgical procedure. The surgery was performed under general anaesthesia, with the patient supine and the head turning to the normal side. Two incision lines were marked on the paralyzed side of the face. To expose the full length of the SCM, the major line started from the crus helicis reaching the earlobe, turned around the mandibular angle until the mastoid process, and then was extended downward along the posterior border of the SCM to the sternoclavicular joint. To expose the orbicularis oris, an adjunctive line was made along the nasolabial crease until the lower oral commissure.

After lifting the platysma muscle, important structures, including the external jugular vein, great auricular nerve, and transverse cervical nerve, were carefully dissected and preserved.

The trapezoid branch of the accessory nerve was identified and dissected with the aid of a nerve detector. The main trunk of the accessory nerve was then tracked backward to identify its branch to the SCM. It is important to note that the accessory nerve sometimes branches after penetrating into the SCM, requiring adequate local dissection. For the ease of muscle transposition, the branch to the SCM was generally split for $1 \sim 2 \mathrm{~cm}$ from the nerve stem (Figure 1). The muscle was then cut off from its attachment sites to the sternum and the clavicle, and elevated to its mastoid head beneath the cutaneous branches of the cervical plexus and external jugular vein. High-frequency electrocautery was used to control hemorrhage. The occipital branch was preserved to guarantee blood supply, while the minor vessels supplying the lower $2 / 3$ of the muscle were mostly ligated.

An arc-like tunnel was prepared within the subcutaneous plane of the cheek. The SCM passed across the cheek obliquely from the preauricular incision to the nasolabial incision. The proximal fixation was performed above and below the zygomatic arch to the periosteum and the superficial layer of deep temporal fascia with braided 2-0 mattress sutures. After trimming the sternal and clavical heads to a proper length, they were fixed to the upper and lower parts of remnant orbicularis oris respectively. The muscle was stimulated by a bipolar electrode to display the new pull vector. The fixation stitches were then adapted accordingly to achieve a better pull effect. In our experience, overcorrection is necessary to offset the effect of gravity and muscle dystrophy after surgery. Usually, the upper oral corner was lifted enough to expose the cuspid.

The new nasolabial crease was created by attachment of the skin to the subcutaneous tissue and SCM fascia. Before skin closure, the skin surplus was excised, hemostasis carefully controlled, and two drain tubes were inserted at cheek and cervical incisions.

Finally, the donor and recipient sites were covered with dressings and bandages, and a cheek retractor was placed at the mouth corner to resist the pulling force of the contralateral normal side.

Postoperative treatment and rehabilitative training. Patients were hospitalized for 1 week after the procedure. Any movement of the oral commissure activating the SCM in the affected side should be avoided during the first month; therefore, an oral retractor was placed and kept at the oral commissure of the affected side for one month to prevent excessive movements.

The postoperative training started 1 month after surgery when the patients noticed any movement in the oral commissure upon neck movement and last for 1 year. Patients were encouraged to elicit oral commissure movement by moving their head initially and then proceed by not moving the head but feeling the contraction of the SCM. Briefly, the training consisted of 4 steps: i) triger targeting: patients were encouraged to move their neck in different directions to find out the most effective trigger of the transferred SCM; ii) muscle power training: patients were encouraged to elicit muscle 

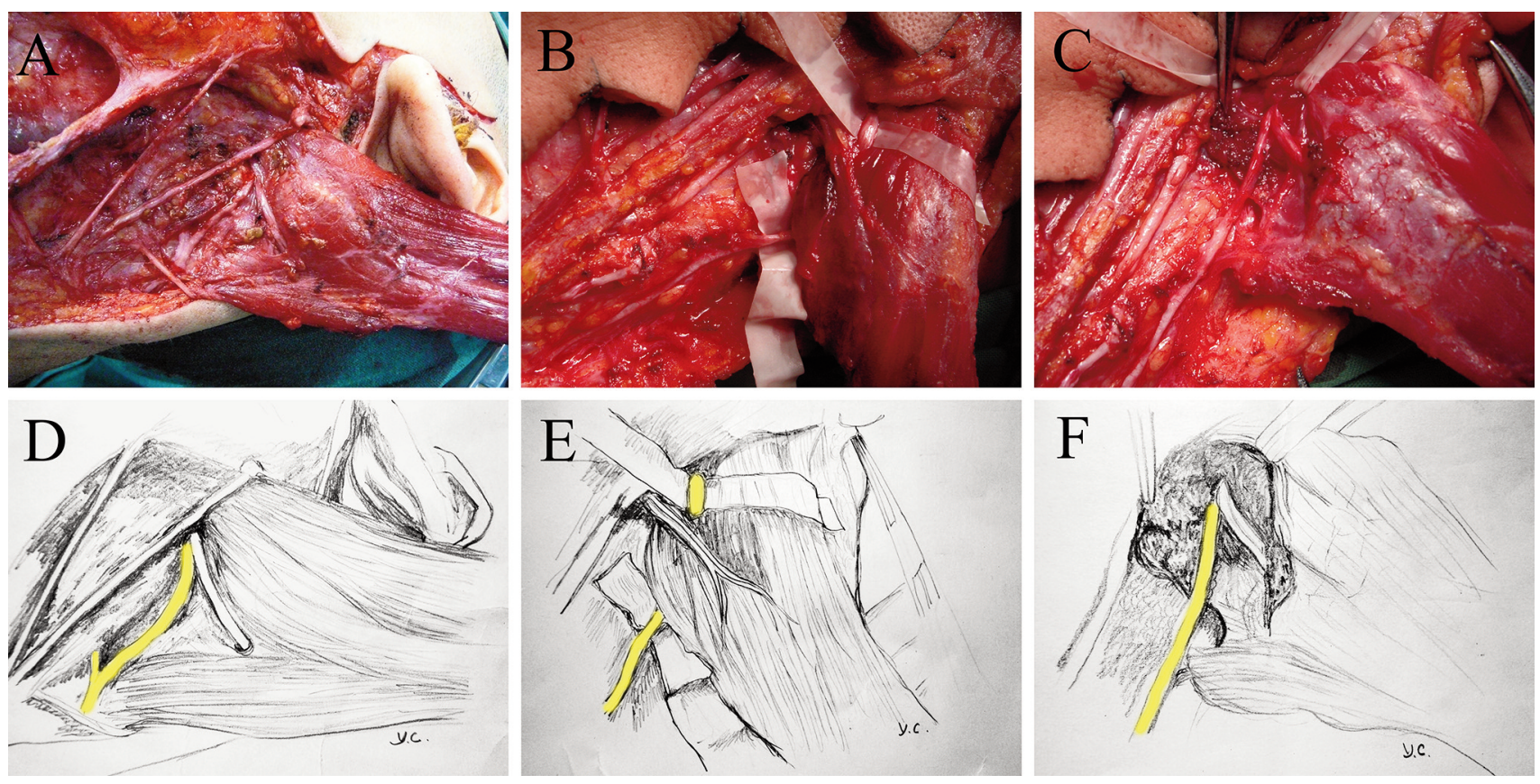

Figure 1. Two different branching patterns of the trapezoid branch (above, intraoperative photo; below: schematic paintings): left row, the trapezoid branch travels alone without entering the SCM, $n=15 / 42$, 35.7\% ( $A, D$ ); part of the trapezoid branch travels inside the SCM together with the SCM branch, $n=27 / 42,64.3 \%(B, E)$; part of the SCM is incised to free the trapezoid branch $(C, F)$.

Table I. Demographic data of the patients.

\begin{tabular}{lcc}
\hline & Mean & Range \\
\hline Age (years) & 37 & $18 \sim 66$ \\
Gender: & & \\
$\quad$ Male & 14 & \\
$\quad$ Female & 28 & $2 \sim 48$ \\
Denervation time (months) & 5 & $12-96$ \\
Follow-up duration (months) & 26 & \\
\hline
\end{tabular}

contraction by moving their neck initially and once strong muscle contraction was acquired, they were encouraged to elicit muscle contraction by not generating obvious neck movements; iii) symmetry training: smiling symmetry was initially trained with the help of a mirror and then without mirror; iv) establishment of conditioned reflex: patients were required to repeat the volitional smile for 20 to $30 \mathrm{~min}$ (in total) each day during the first year after the procedure and use the new vector each time they smile in everyday life.

Methods of outcomes evaluation and statistical analysis. Postoperative follow-up was planned at 3 months, 6 months and every 6 months after that, for at least 12 months. Pre and post-operative pictures and videos were taken to document different expressions, at each follow-up visit. Each patient was evaluated with HouseBrackmann (HB) scale by a third physician who did not participate in any of the operative procedures and was blind to information as
Table II. Etiologies of facial paralysis in the patients included in the study.

\begin{tabular}{lc}
\hline Etiology & Number of patients \\
\hline Cholesteatoma & 2 \\
Facial nerve schwannoma & 2 \\
Angioma & 2 \\
Trauma & 3 \\
Acoustic neuroma & 21 \\
Bell's palsy & 5 \\
Otitis media & 2 \\
Congenital & 1 \\
Virus infection & 1 \\
Stroke & 1 \\
Parotid gland tumor & 2 \\
Total number of patients & 42 \\
\end{tabular}

to when the photographs are taken. Facial symmetry and oral commissure excursion was analyzed with Facegram (V1, MEEI). Symmetry ratio was calculated as ( $\mathrm{C}$ value in the affected side/ $\mathrm{C}$ value in the unaffected side $) \times 100 \%$. Oral commissure excursion was equal to $\mathrm{C}$ value at smile minus $\mathrm{C}$ value at rest.

All data collected was coded so that no patient identification was possible. Descriptive statistics such as mean, standard deviation, and percentage were used to present the data. Comparisons between preoperative and postoperative measurements were performed by using paired $t$ tests. The significance level was set at $p<0.05$. 

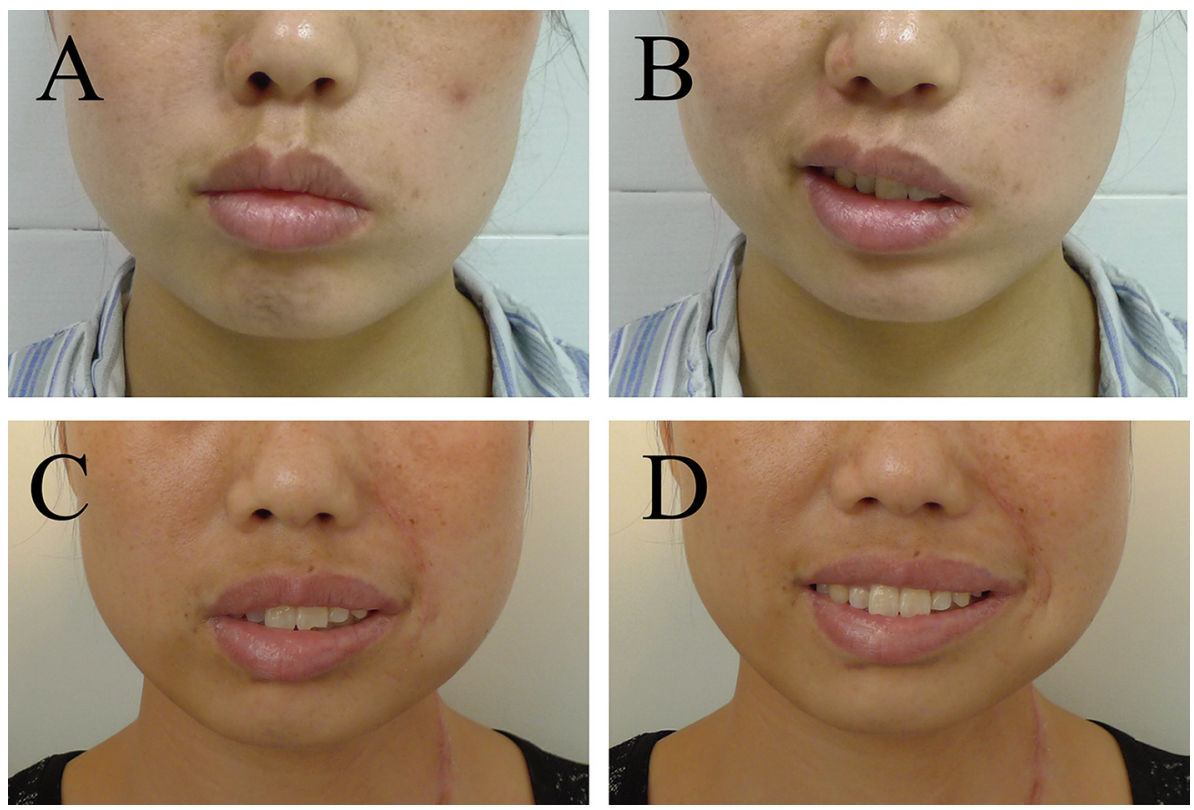

Figure 2. Pre-operative $(A, B)$ and 7 months post-operative $(C, D)$ views of a 34-year old female patient. Left: patient at rest; Right: patient smiling.

\section{Results}

Demographics and clinic pathological data of the patients. From January 2009 to December 2015, a total number of 51 patients with long-standing facial palsy or frozen faces underwent facial reanimation with SCM transfer. Among them, 42 patients treated met inclusion criteria. All procedures were performed by the same surgeon (C.Y.). The mean age of the patients at the time of surgery was 37 years (range $=18-66$ ) with a mean denervation time of 5 years (range $=2-48$ ) (Table I). The mean follow-up time was 26 months (range=12-96). The etiologies of facial palsy were acoustic neuroma extirpation $(n=21)$, cholesteatoma resection $(n=2)$, parotid tumor resection $(n=2)$, Bell's palsy $(n=5)$, otitis media $(n=2)$, angioma $(n=2)$, facial nerve schwannoma $(n=2)$, trauma $(n=2)$, congenital $(n=1)$, virus infection $(n=1)$, stroke $(n=1)$ (Table II).

Anatomy of the accessory nerve branches. Two different patterns of the trapezoid muscle branch of the accessory nerve were encountered during the procedure (Figure 1). In 27 out of 42 cases $(64.3 \%)$, the trapezoid branch traveled through the SCM together with the SCM branch (Figure 1B, $1 \mathrm{E}$ ), which would require partial section of the SCM (Figure 1C, 1F) to free the trapezoid branch so that the SCM could get more mobility to rotate thoroughly. In the rest of cases (15 of $24,35.7 \%$ ), it traveled alone without entering the SCM (Figure 1A, 1D), which made the dissection much easier.

Functional outcome. The contraction of the transferred SCM was first observed at 3 months after the procedure in the majority of the patients $(n=40)$. Maximal contraction and symmetric smile were achieved in all patients at 6 months after the procedure (Figure 2, Figure 3 and Figure 4).

The HB score decreased significantly from the preoperative value of 4.7 to the 12-month postoperative 3.6 $(p<0.05$, Figure 5). The Facegram analysis (Table III) revealed a significantly improved symmetry both at smile ( 0.52 pre-operative $v s .1 .02$ post-operative, $\mathrm{C}$ value of the affected side at smile/C value of contralateral normal side at smile, $p<0.05)$ and at repose $(0.76$ pre-operative vs. 0.99 post-operative, $\mathrm{C}$ value of the affected side at repose/ $\mathrm{C}$ value of the contralateral normal side at repose, $p<0.05)$. A significant increase in terms of oral commissure excursion in the affected side was also observed 12 months after the procedure $(-3.02 \mathrm{~mm}$ pre-operative $v s .5 .93 \mathrm{~mm}$ postoperative, $p<0.05$, Table IV). No spontaneous smile was observed in any patient.

Complications. Contour deformity in the donor SCM area of the neck was observed in all patients and was considered disturbing in two patients. The scar was visible in all cases but no severe hypertrophic scar was observed at follow-up (Figure 6).

No muscle necrosis nor functional compromise at neck or the shoulder joints were recorded in any of the patients.

\section{Discussion}

Longstanding facial palsy can be reanimated by either free muscle flaps transplantation or local muscle flaps transfer 

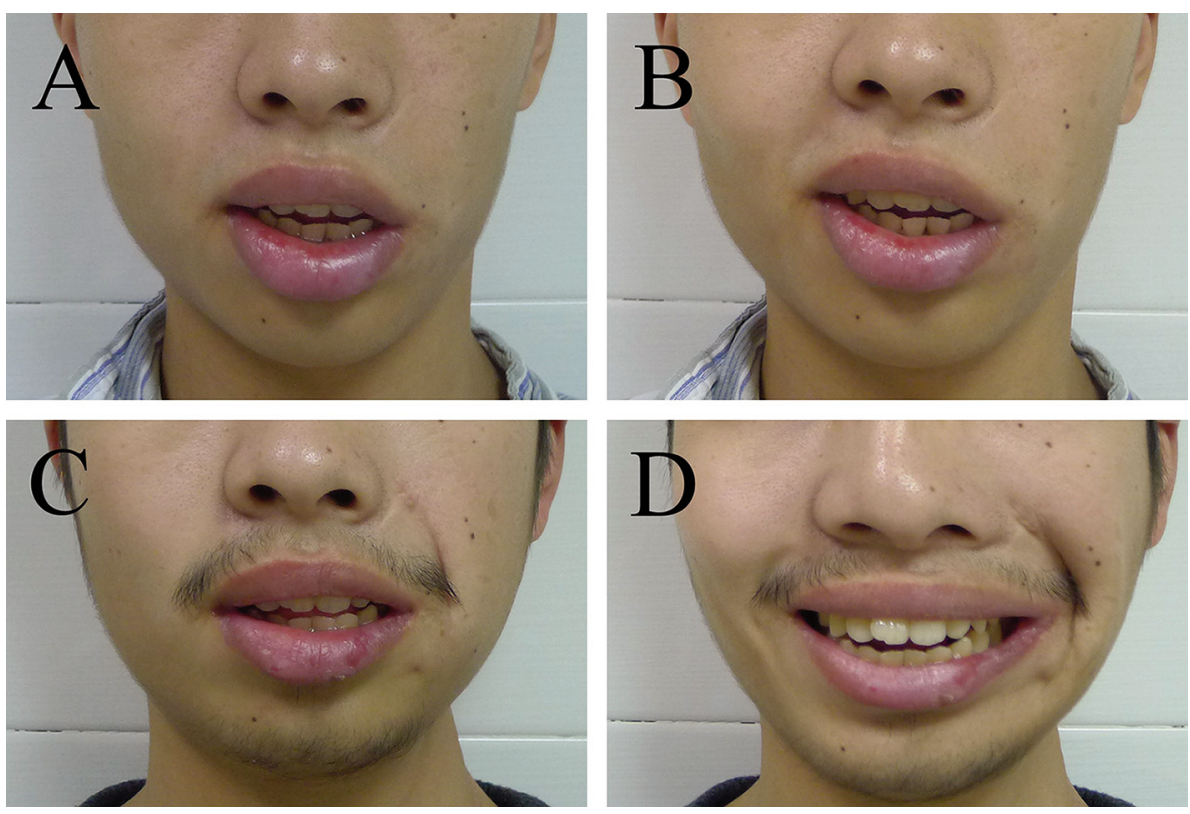

Figure 3. Pre-operative $(A, B)$ and 6 months post-operative $(C, D)$ views of an 18-year old male patient. Left: patient at rest; right: patient smiling.
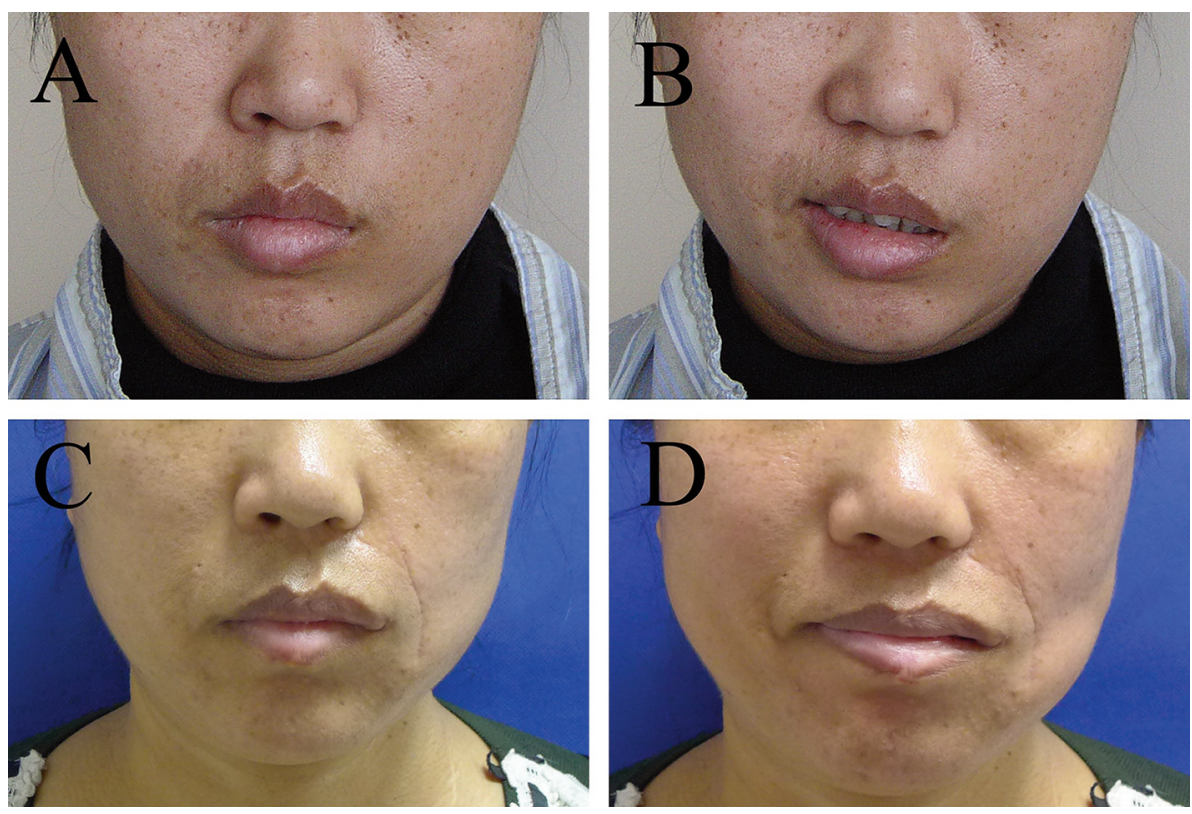

Figure 4. Pre-operative $(A, B)$ and 9 years post-operative $(C, D)$ views of a 42 -year old female patient. Left: patient at rest; right: patient smiling.

$(10,11,21)$. The free muscle flaps are able to generate stronger muscle contraction and better oral commissure excursion (22). Moreover, they can provide the patients with good synchronicity and spontaneity when coaptated with the contralateral facial nerve with cross facial nerve graft or a long nerve pedicle (22). Nevertheless, these advantages are also accompanied with the higher risks associated with blood vessel anastomosis and longer surgical time.

Local muscle flap (mini-temporalis and lengthening temporalis myomectomy), on the other hand, are less invasive and time consuming, but generate limited oral commissure excursion in comparison to free muscle flaps. However, in experienced hands, the lengthening temporalis 

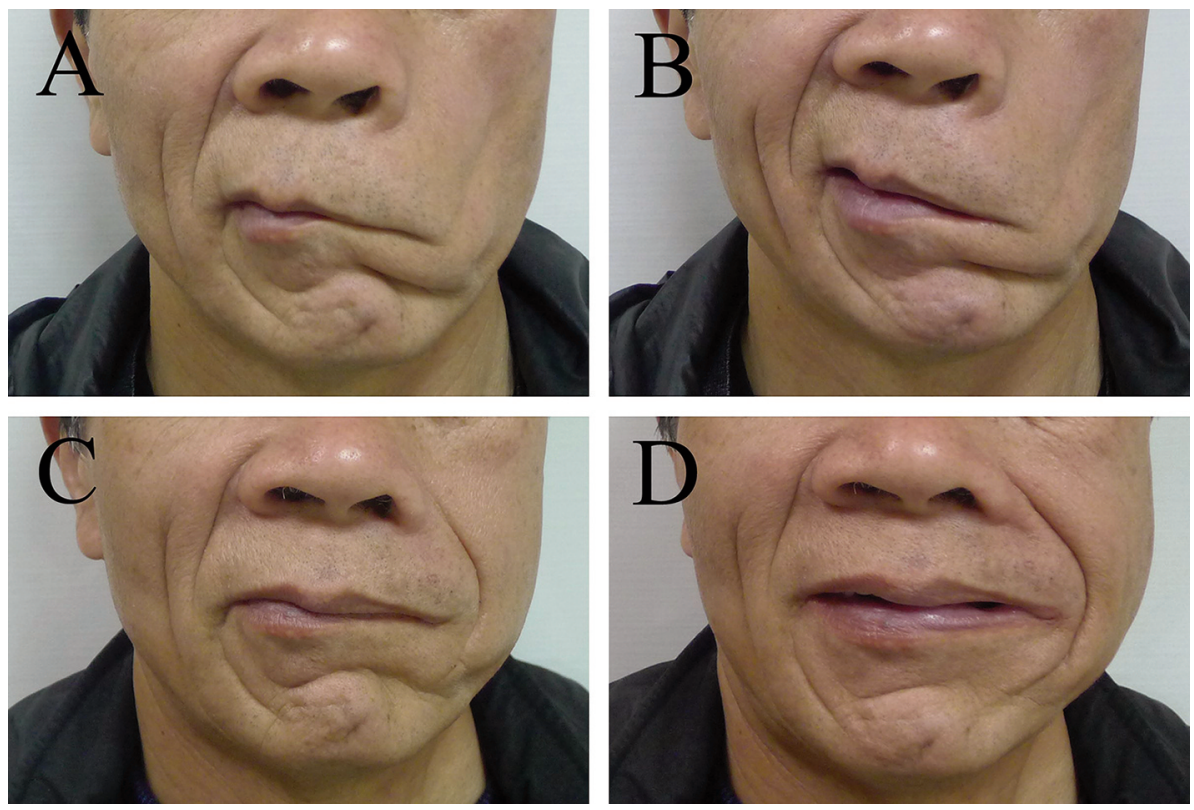

Figure 5. Pre-operative $(A, B)$ and 10 months post-operative $(C, D)$ views of a 66-year old man. Left: patient at rest; right: patient smiling.

Table III. Oral commissure symmetry at repose and smile (c value, $\mathrm{mm}$ ).

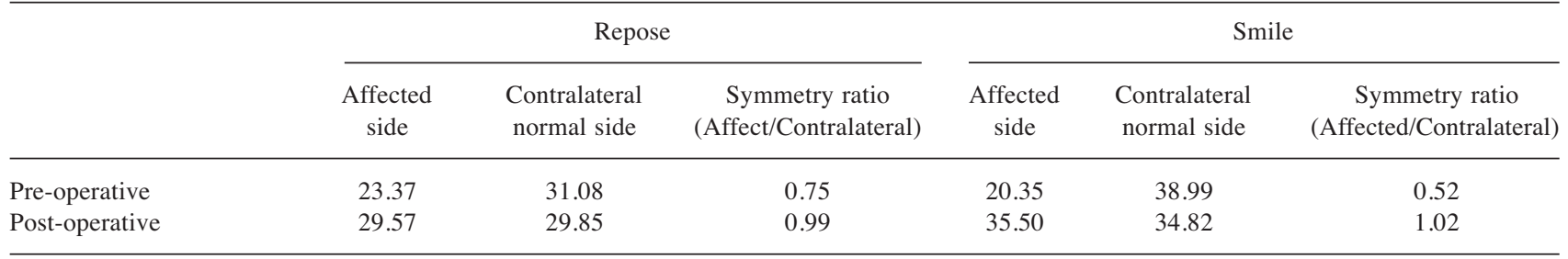

myomectomy, is able to guarantee satisfactory results $(15$, 23). The use of both local temporalis muscle flap and free muscle flaps innervated by masseter nerve requires intact function of the trigeminal nerve, which in many cases of facial palsy patients can be compromised in by intracranial tumor resection. In this case, free flaps with other donor nerve, e.g., hypoglossal nerve or the accessory nerve need to be considered $(9,24-26)$. Therefore, in addition to personal preferences of the surgeons and the patients, the choice of the surgical procedure also depends on the conditions of donor and recipient sites.

Our group previously described a technique based on the use of the local SCM muscle flap (16). This was introduced in our center in the early 1980 s by Dr. CY, and has been performed in over 300 cases. In our current study of 42 cases, oral commissure movement was documented in all patients at their last follow-up visit. Therefore, this technique provides a valuable alternative for facial reanimation with local muscle
Table IV. Oral commissure excursion.

\begin{tabular}{lcc}
\hline & Pre-operative $(\mathrm{mm})$ & Post-operative $(\mathrm{mm})$ \\
\hline Contralateral normal side & 7.9 & 5.7 \\
Affected side & -3.05 & 5.9 \\
\hline
\end{tabular}

flap, especially for patients with compromised trigeminal nerve function and those who are not good candidates for free muscle flap transfer. Previous attempt to use the pedicled SCM muscle flap for facial reanimation were reported by Schottstaedt Larsen and Bost in 1955 and was based on their work in substitution of the masseter with the SCM muscle. They severed the origin and insertion of the muscle and fixed one end to the temporal area and parotid gland and the other end to the oral commissure (16). This attempt failed due to the necrosis of the flap. Conway commented in 1958 that the 

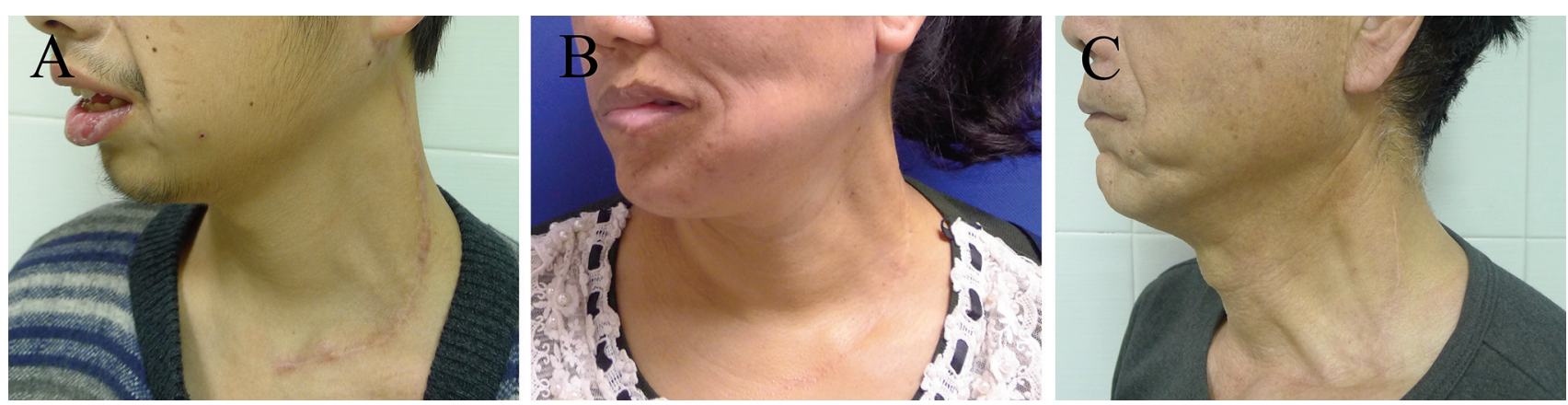

Figure 6. Post-operative scars in an 18-year old male patient (A, 6 months post-operative), and a 42-year old female patient (B, 9 years postoperative) and a 66-year old man (C, 10 months post-operative).

SCM muscle is not a good candidate muscle flap for facial reanimation because the pedicle is too short, which could easily result in a compromised blood supply and eventually necrosis after the transfer. He also mentioned that the bulky volume of the muscle and the direction of muscle contraction are not optimal (27).

However, with a better understanding of the anatomy (18, $28,29)$, the SCM flap was frequently used to avoid the Frey's syndrome after excision of parotid gland tumor because of its vicinity and the easy rotation of the flap to the parotid region without additional incisions $(19,20)$. The flap is long enough to cover all the branches of the facial nerve and it also decreases the depression of the surgical area after parotid gland resection. In addition, there is a low risk of necrosis of the flap with a better understanding of its vascularization, and there is a low risk of complications of, primarily, spinal accessory nerve injury $(19,20)$.

The blood supply for the SCM muscle is segmental, with blood vessels arising from the occipital artery, the superior thyroid artery and the suprascapular artery (18). With our technique, in opposition to the pedicled free SCM flap described by Schottstaedt Larsen and Bost, blood vessels arising from the occipital artery were protected within the muscle bundles by avoiding extensive dissection. Blood vessels from the superior thyroid artery were sacrificed to allow the mobility of the muscle flap. This ensured a secure blood supply from the superior pedicle, which was protected by the muscle fibers to avoid extensive tension after transfer of the muscle.

Another key point for this procedure is to guarantee a proper direction of the vector for the oral commissure excursion. The SCM muscle originates from the mastoid process, which is almost in the same horizontal line with the oral commissure. Therefore, it is crucial to anchor the muscle higher than the oral commissure in the zygomatic arch with braided 2-0 mattress sutures in order to mimic the native vector.

Our SCM transfer also resulted in strong lateral excursion of the oral commissure $(5.93 \mathrm{~mm})$, which is not as strong as free muscle flap (10.4 mm), but comparable to the result of masseter nerve transfer $(6.8 \mathrm{~mm})$ and stronger than that of the temporalis myoplasty $(3.1 \mathrm{~mm})(30)$. This is probably due to the superiority in the quantity of muscle fibers and undisturbed innervation from the accessory nerve, which also enables the patients to gain function in the early stages after the procedure. In the meanwhile, the vector orientation might also play a role, as the lateral movement of the oral commissure generated by contraction of the lower part of the muscle is efficient in balancing the oral commissure movement symmetry. Symmetric smile could be achieved after 3-6 months of training. Most patients were able to smile without turning or moving their heads. Spontaneous smile was reported in patients reanimated with masseter nerve transfer, which was probably a result of adaptation in the cerebral plasticity. This phenomenon, however, was not found in any of our cases.

Due to the vicinity of the SCM to the oral commissure and its well-studied anatomy, it is not complicated for a surgeon to conduct this procedure and it is obviously less time consuming when compared with free muscle flap. In addition, due to the abundance of the muscle fibers in the SCM and undisturbed innervation of the accessory nerve, it is not difficult to have stable results with strong muscle contraction when compared to other local muscle flap techniques. In addition, SCM is mainly composed of fast fibers and complex fibers (31), which, in contrary to mostly slow fibers in limb muscles, is very similar to that of the mimetic muscles and could also contribute to functional recovery.

Early bulkiness caused by transfer of the muscle is observed in all patients. However, significant improvement was found one year after the procedure with a reduction of almost half of the original volume caused by atrophy despite the undisturbed innervation from the accessory nerve. This could be due to the redundant innervation of the SCM muscle from multiple nerve branches in addition to the accessory nerve, such as nerve fibers from the cervical 
branches or in some cases the facial nerves $(32,33)$, which could be severed during elevation of the muscle flap.

No functional compromise in the neck was noted. The scar in the nasolabial fold and the preauricular region were not obvious. This, however, could be optimized in the future with an endoscopic harvesting of the muscle through a facelift incision, which would make the scar well-hidden. Asymmetry in neck contour was noted but was not considered disturbing in all patients except two.

Post-operative rehabilitative training is important for our patients as the nerve supply for this vector is from the accessory nerve instead of the facial nerve. This will require efforts from both patients and surgeons. Therefore, expected incompliance with the post-operative training should be regarded as contraindication for this procedure. Patients with extremely wide face or short neck should also be excluded as the length of the SCM might not be long enough for the transfer and the bulkiness of the flap could be relatively excessive in these patients.

Compared to previous works on the subject, our technique optimizes protection of both vascular and neural supply to ensure the functionality of the transferred muscle flap. A notfunctional flap could be disastrous for facial reanimation but acceptable for wound coverage. The major contribution of our method is the identification of the two patterns of nerve supply to the muscle and the presentation of subsequent surgical strategy for each situation. In particular, our technique requires partial section of the SCM only in case of trapezoid branch traveling through the SCM together with the SCM branch to allow easy flap rotation.

In conclusion, the use of the SCM muscle flap for treatment of longstanding facial palsy presents the advantage of transferring a local muscle flap providing early recovery and strong muscle contraction. This is not a complicated or time-consuming procedure and guarantees good results in most cases. It should be considered as an option for patients who are not good candidates for free muscle flap transplantation, especially for those with concomitant compromise of the trigeminal nerve. However, due to the various causes of the facial palsy, a careful preoperative evaluation should be conducted, especially in patients with severe vascular formation or who had ablative surgeries for tumors as the success of this procedure depend on safe and reliable blood supply of the SCM.

\section{Conflicts of Interest}

The Authors declare no conflicts of interest.

\section{Authors' Contributions}

Study design: Wenjin Wang, Yizuo Cai, Carlo M. Oranges, Chuan Yang, and Wei Li; Data collection: Yizuo Cai; Data analysis: Wenjin
Wang, Yizuo Cai, Carlo M. Oranges, Chuan Yang, and Wei Li; Manuscript drafting: Wenjin Wang; Critical revision: all Authors. Final approval: all Authors.

\section{Acknowledgements}

This study was supported by Natural Science Foundation of Shanghai, 16ZR1419800, 18411961800; Shanghai Jiao Tong University multidisciplinary project, YG2016MS10; Shanghai Municipal Science and Technology Commission Fund, 18411961800.

\section{References}

1 Jowett $\mathrm{N}$ and Hadlock TA: A Contemporary approach to facial reanimation. JAMA Facial Plast Surg 17(4): 293-300, 2015. PMID: 26042960. DOI: 10.1001/jamafacial.2015.0399

2 Li MK, Niles N, Gore S, Ebrahimi A, McGuinness J and Clark JR: Social perception of morbidity in facial nerve paralysis. Head Neck 38(8): 1158-1163, 2016. PMID: 27225347. DOI: 10.1002/hed.24299

3 Goines JB, Ishii LE, Dey JK, Phillis M, Byrne PJ, Boahene KD and Ishii M: Association of facial paralysis-related disability with patient- and observer-perceived quality of life. JAMA Facial Plast Surg 18(5): 363-369, 2016. PMID: 27253905. DOI: 10.1001/jamafacial.2016.0483

4 Ryu NG, Lim BW, Cho JK and Kim J: Quality of life differences in patients with right- versus left-sided facial paralysis: Universal preference of right-sided human face recognition. J Plast Reconstr Aesthet Surg 69(9): e197-e203, 2016. PMID: 27449749. DOI: 10.1016/j.bjps.2016.06.021

5 Wang W, Yang C, Li W, Li Q and Zhang Y: Masseter-to-facial nerve transfer: is it possible to rehabilitate the function of both the paralyzed eyelid and the oral commissure? Aesthetic Plast Surg 36(6): 1353-1360, 2012. PMID: 23052376. DOI: 10.1007/ s00266-012-9951-9

6 Park H, Jeong SS and Oh TS: Masseter nerve-based facial palsy reconstruction. Arch Craniofac Surg 21(6): 337-344, 2020. PMID: 33663141. DOI: 10.7181/acfs.2020.00682

7 Wang W, Yang C, Li Q, Li W, Yang X and Zhang YX: Masseterto-facial nerve transfer: a highly effective technique for facial reanimation after acoustic neuroma resection. Ann Plast Surg 73 Suppl 1: S63-S69, 2014. PMID: 25115378. DOI: 10.1097/SAP. 0000000000000246

8 Negley KJ, Rasool A and Byrne PJ: Motor relearning after hypoglossal-facial nerve anastomosis. Am J Phys Med Rehabil 100(6): e85-e88, 2021. PMID: 32796157. DOI: 10.1097/ PHM.0000000000001562

9 Ebersold MJ and Quast LM: Long-term results of spinal accessory nerve-facial nerve anastomosis. J Neurosurg 77(1): 5154, 1992. PMID: 1607971. DOI: 10.3171/jns.1992.77.1.0051

10 Guerreschi P and Labbe D: Lengthening temporalis myoplasty: a surgical tool for dynamic labial commissure reanimation. Facial Plast Surg 31(2): 123-127, 2015. PMID: 25958897. DOI: 10.1055/s-0035-1549039

11 Chen G, Yang X, Wang W and Li Q: Mini-temporalis transposition: a less invasive procedure of smile restoration for long-standing incomplete facial paralysis. J Craniofac Surg 26(2): 518-521, 2015. PMID: 25759922. DOI: 10.1097/SCS. 0000000000001522 
12 Watanabe Y, Yamamoto T, Hirai R, Sasaki R, Agawa K and Akizuki T: One-stage free transfer of latissimus dorsi-serratus anterior combined muscle flap with dual innervation for smile reanimation in established facial paralysis. J Plast Reconstr Aesthet Surg 73(6): 1107-1115, 2020. PMID: 32334999. DOI: 10.1016/j.bjps.2020.01.032

13 Hontanilla B, Marre D and Cabello Á: Facial reanimation with gracilis muscle transfer neurotized to cross-facial nerve graft versus masseteric nerve: a comparative study using the FACIAL CLIMA evaluating system. Plast Reconstr Surg 131(6): 12411252, 2013. PMID: 23416438. DOI: 10.1097/PRS. 0b013e31828bd4da

14 Hembd A, Harrison B, Rocha CSM, Rocha FS, Chamseddin K, Labbé D, Cárdenas-Mejía A and Rozen SM: Facial reanimation in the seventh and eighth decades of life. Plast Reconstr Surg 141(5): 1239-1251, 2018. PMID: 29697623. DOI: 10.1097/ PRS.0000000000004329

15 Nduka C, Hallam MJ and Labbe D: Refinements in smile reanimation: 10-year experience with the lengthening Temporalis Myoplasty. J Plast Reconstr Aesthet Surg 65(7): 851-856, 2012. PMID: 22445694. DOI: 10.1016/j.bjps.2012.02.006

16 Yang C, Wang W and Zhang Q: [The restoration of chronic facial paralysis with a modified technique of sternocleidomastoid muscle transposition]. Zhonghua Zheng Xing Wai Ke Za Zhi 21(2): 104-106, 2005. PMID: 16011194.

17 Sokcic A: [Surgical treatment of facial paralysis]. HNO 6(4): 104-107, 1957. PMID: 13428139.

18 Leclère FM, Vacher C and Benchaa T: Blood supply to the human sternocleidomastoid muscle and its clinical implications for mandible reconstruction. Laryngoscope 122(11): 2402-2406, 2012. PMID: 23007956. DOI: 10.1002/lary.23430

19 Sanabria A, Kowalski LP, Bradley PJ, Hartl DM, Bradford CR, de Bree R, Rinaldo A and Ferlito A: Sternocleidomastoid muscle flap in preventing Frey's syndrome after parotidectomy: a systematic review. Head Neck 34(4): 589-598, 2012. PMID: 21472880. DOI: 10.1002/hed.21722

20 Grosheva M, Horstmann L, Volk GF, Holler C, Ludwig L, Weiß V, Finkensieper M, Wittekindt C, Klussmann JP, GuntinasLichius $\mathrm{O}$ and Beutner D: Frey's syndrome after superficial parotidectomy: role of the sternocleidomastoid muscle flap: a prospective nonrandomized controlled trial. Am J Surg 212(4): 740-747.e1, 2016. PMID: 27083066. DOI: 10.1016/j.amjsurg. 2016.01.020

21 Terzis JK and Olivares FS: Use of mini-temporalis transposition to improve free muscle outcomes for smile. Plast Reconstr Surg 122(6): 1723-1732, 2008. PMID: 19050524. DOI: 10.1097/ PRS.0b013e31818a9adc

22 Bos R, Reddy SG and Mommaerts MY: Lengthening temporalis myoplasty versus free muscle transfer with the gracilis flap for long-standing facial paralysis: A systematic review of outcomes. J Craniomaxillofac Surg 44(8): 940-951, 2016. PMID: 27364924. DOI: 10.1016/j.jcms.2016.05.006
23 Aljudaibi N, Bennis Y, Duquennoy-Martinot V, Labbé D and Guerreschi P: Lengthening temporalis myoplasty: Virtual animation-assisted technical video. Plast Reconstr Surg 138(3): 506e-509e, 2016. PMID: 27556626. DOI: 10.1097/PRS. 0000000000002512

24 Hontanilla B and Aubá C: Smile reconstruction through bilateral muscular transplants neurotized by hypoglossal nerves. J Craniofac Surg 22(3): 845-847, 2011. PMID: 21558933. DOI: 10.1097/SCS.0b013e31820f7d50

25 Hammerschlag PE: Facial reanimation with jump interpositional graft hypoglossal facial anastomosis and hypoglossal facial anastomosis: evolution in management of facial paralysis. Laryngoscope 109(2 Pt 2 Suppl 90): 1-23, 1999. PMID: 10884169. DOI: 10.1097/00005537-199902001-00001

26 Chuang DC, Lu JC and Anesti K: One-stage procedure using spinal accessory nerve (XI)-innervated free muscle for facial paralysis reconstruction. Plast Reconstr Surg 132(1): 117e-129e, 2013. PMID: 23806931. DOI: 10.1097/PRS.0b013e318290f8cd

27 Conway H: Muscle plastic operations for facial paralysis. Ann Surg 147(4): 541-552, 1958. PMID: 13521669. DOI: 10.1097/ 00000658-195804000-00010

28 Alagöz MS, Cağri Uysal A, Tüccar E and Sensöz O: How cranial could the sternocleidomastoid muscle be split? J Craniofac Surg 16(2): 201-204, 2005. PMID: 15750415. DOI: 10.1097/00001665-200503000-00004

29 Saha A, Mandal S, Chakraborty S and Bandyopadhyay M: Morphological study of the attachment of sternocleidomastoid muscle. Singapore Med J 55(1): 45-47, 2014. PMID: 24241357. DOI: $10.11622 /$ smedj.2013215

30 Harii $\mathrm{K}$, Ohmori $\mathrm{K}$ and Torii S: Free gracilis muscle transplantation, with microneurovascular anastomoses for the treatment of facial paralysis. A preliminary report. Plast Reconstr Surg 57(2): 133-143, 1976. PMID: 1250883. DOI: 10.1097/ 00006534-197602000-00001

31 Cvetko E, Karen $\mathrm{P}$ and Eržen I: Myosin heavy chain composition of the human sternocleidomastoid muscle. Ann Anat 194(5): 467-472, 2012. PMID: 22658700. DOI: 10.1016/ j.aanat.2012.05.001

32 Blythe JN, Matharu J, Reuther WJ and Brennan PA: Innervation of the lower third of the sternocleidomastoid muscle by the ansa cervicalis through the $\mathrm{C} 1$ descendens hypoglossal branch: a previously unreported anatomical variant. Br J Oral Maxillofac Surg 53(5): 470-471, 2015. PMID: 25747248. DOI: 10.1016/ j.bjoms.2015.01.005

33 Cvetko E: Sternocleidomastoid muscle additionally innervated by the facial nerve: case report and review of the literature. Anat Sci Int 90(1): 54-56, 2015. PMID: 24347311. DOI: $10.1007 /$ s12565-013-0224-8

Received August 5, 2021

Revised November 20, 2021

Accepted November 26, 2021 